Article

\title{
Statistical Ensemble Theory of Gompertz Growth Model
}

Takuya Yamano ${ }^{1,2}$

${ }^{1}$ Department of Information Sciences, Faculty of Science, Kanagawa University, 2946 Tsuchiya, Hiratsuka, Kanagawa 259-1293, Japan

${ }^{2}$ Department of Physics, Ochanomizu University, 2-1-1 Otsuka, Bunkyo-ku, 112-8610, Tokyo, Japan;

E-Mail: yamano@amy.hi-ho.ne.jp

Received: 1 August 2009 / Accepted: 2 November 2009 / Published: 5 November 2009

\begin{abstract}
An ensemble formulation for the Gompertz growth function within the framework of statistical mechanics is presented, where the two growth parameters are assumed to be statistically distributed. The growth can be viewed as a self-referential process, which enables us to use the Bose-Einstein statistics picture. The analytical entropy expression pertain to the law can be obtained in terms of the growth velocity distribution as well as the Gompertz function itself for the whole process.
\end{abstract}

Keywords: Gompertz function; tumour growth; Bose-Einstein statistics; entropy

\section{Introduction}

Growth phenomena are universal in nature and their underlying governing laws have attracted considerable attention from many researchers. We shall focus on one of these laws in this paper, namely that of Gompertzian evolution [1]. The Gompertz law was first used to describe the tumour growth phenomena [2], where the mammalian tumour growth data were fitted with the Gompertz function. Here we go beyond the original context of tumour growth and our results should be applicable to systems that simply obey the Gompertz model. [3] should be consulted for both a historical and pedagogical review on the use of the Gompertz growth function in the context of tumour growth.

A justification for the validity of the application of the Gompertz function to tumour growth is still lacking. However, it is found empirically that the dynamics of tumour growths follows $\exp [a(1-\exp [-b t])]$, where $t$ is time, $a$ and $b$ are parameters which are determined by fitting experimental data. The interpretation of the Gompertz function has been attempted in various fields 
such as biology, mathematics and thermodynamics (for the examples, see [3]). Some papers have focused on the issues on where the law comes from. For example, the derivation of the functional form based on the self-similar process [4], on the combinatorial consideration of the degree of cellular heterogeneity [5], and on the change of entropy for the growth process [6] have all been suggested. The statistical mechanics for the Gompertz model whose system consists of interacting species were considered more than two decades ago [7,8]. Recent developments include thermodynamics considerations and a stochastic model. The thermodynamics approach considered an energy balance among the different cell activities at some fixed time [9] and a stochastic model incorporating environmental fluctuations was investigated in [10]. These attempts have much to do with statistical description for the tumour growth phenomena and are the main motivation behind this work. Furthermore, the fractal nature of the temporal and spatial process of Gompertz dynamics [11] and the fractal-stochastic duality as a consequence of the intercellular interaction [12] has also been investigated.

In spite of the above studies and interests in the Gompertz function itself, a statistical ensemble approach to the model is still lacking. This is probably due to the broad range of applications of the Gibbs ensemble theory in equilibrium statistical mechanics whose form is exponential and also due to the usefulness for curve fittings with two parameters tuning. Main aims of this paper are to focus on the theoretical aspect of Gompertz growth and especially to investigate several ensemble descriptions for the distribution functions whose growth parameters differ by some "elements". By "elements" we suppose that whole organism consists of clusters or ensemble of the tumour tissue that has a pair of different proliferation rates $a_{i}$ and $b_{i}$, where $i$ discriminates individual tissue. We may also consider the set of tumour formed in different times in different parts of an organ. We are motivated from a view point of statistical mechanics to describe these situations statistically.

The organization of this paper is as follows. We consider the functional form of Gompertz as a consequence of a self-reference process in the distribution function in the next section. In Section 3, we deal with properties of fluctuations of a growth rate in order to see the statistical nature. Section 4 revisits the issue of entropy properties by defining it differently from one used in previous works in the literature. A demonstration of the statistical mechanics formulation of the Bose-Einstein statistics will be provided in Section 5. Summary and concluding remarks in Section 6 close this paper.

\section{Distributions Generated from a Self-referential Process}

To understand the Gompertz growth process from a view point of self-generating phenomena of tumour cells, which can be attributed to complex intercellular interactions, we consider a feedback process at the level of distribution, where the process is assumed to proceed in "self-referential" way. By "self-referential", we mean both replication and a feedback process as we shall explain later. In this approach, the original distribution of the number of cells $f(t)$ derives a distribution via a proliferation rate $r(t)$ multiplicatively. It is defined

$$
F(t)=f(t) \pm r(t) F(t)
$$

where $F(t)$ represents the derived distribution of cells generated from an underlying dynamical process with a given initial distribution. We interpret the above relation in the following way. That is, $F(t)$ is defined by r.h.s at equal-time. The number of tumour cells at time $t$ is given by $f(t)=f_{0} r(t)$, where $f_{0}$ 
is the number of initial cells and therefore $r(0)=1$. In the case of the upper sign (+) in Equation (1), it is referred to as self-replication process [13], on the other hand, when the process proceeds according to the lower sign (-), we call it self-feedback process. It follows from the above definition that the distribution $F(t)$ is produced from the original distribution $f(t)$ as

$$
F(t)=\frac{r(t)}{1 \mp r(t)} f_{0}
$$

What is the physical meaning of $F(t)$ ? As $f(t)$ can represent the number of elements, $F(t)$ can have a characteristic of the cumulative number of them up to time $t$, because Equation (2) expresses the sum of infinite series with the geometric ratio $r$ (for $(-)$ sign: $(0<r<1)$, for $(+)$ sign: $r<-1)$ and the first term $r f_{0}$. Moreover, we can regard that the self-replication process relates the two distributions $-F(t) / f_{0}$ and $f(t) / f_{0}$ by the Euler's transform [13]. Under this transform between $-F(t) / f_{0}$ and $f(t) / f_{0}, F(t)$ contains information of all the elements generated in the process, where the original element at $t=0$ produces accompanying ones. Again, it is an interpretation of Equation (2). In the self-replication process, the derived distribution $F(t)$ has a singularity at $t=0$. On the other hand, in the case of the self-feedback process, the evolution starts with the half of the initial population and the new distribution is produced by subtraction of the new one multiplied by the growth rate from the original distribution (negative feedback). The interesting application of these processes is performed for the Gompertz growth model

$$
f(t)=f_{0} e^{a\left(1-e^{-b t}\right)}
$$

and the growth factor is therefore $r(t)=e^{a\left(1-e^{-b t}\right)}$, which provides the following form,

$$
F(t)=\frac{1}{e^{-a\left(1-e^{-b t}\right)} \mp 1} f_{0}
$$

Therefore, in short time region or for small growth rate $b$, which enables us to regard $b t \ll 1, F(t)$ can be approximated, by the series expansion of $e^{-b t}$, as

$$
F(t) \sim \frac{1}{e^{-a b t} \mp 1} f_{0}
$$

An interesting point in Equation (5) is that $F(t)$ takes the logistic function in the positive time regime for $a>0$ when the process is self-replicating. The logistic model has been used for chemotherapy optimization [14] and used for describing immune response to tumour [15]. It would be also worthwhile noting that, for $a<0$, the obtained distributions are remarkably similar to distributions in the Bose-Einstein and the Fermi-Dirac ones in quantum statistical mechanics in the case of minus and plus signs in Equation (5), respectively. We shall further pursue one of this similarities in Section 5.

Next, we show that the parameter of growth rate $a$ plays a role of the exponent of the power law decay of $F(t)$. We are concerned with the time region where $b t \ll 1$ holds and note the fact that for small values of $x$, the gamma function is expressed as $\Gamma(1+x) \approx \Gamma(1)+x \Gamma^{\prime}(x)=1-\gamma x$, where $\gamma=-\Gamma^{\prime}(1) / \Gamma(1)=0.57721566 \cdots$ is the Euler's constant. Then, the expression for the power of the gamma function $\Gamma(x)^{\xi}=[\Gamma(1+x) / x]^{\xi}$ can be approximated for large values of $\xi$ as

$$
\left(\frac{1-\gamma x}{x}\right)^{\xi} \approx x^{-\xi} e^{-\gamma x \xi}
$$


Therefore, in the range $b t \ll 1$, regarding the variables as $x=b t / \gamma$ and $\xi=a$, then substituting Equation (6) into Equation (2) leads

$$
F(t)=f_{0} \frac{1}{e^{-\gamma x \xi} \mp 1} \approx \frac{f_{0}}{[x \Gamma(x)]^{\xi} \mp 1} \approx f_{0}\left[\left(\frac{b t}{\gamma}\right) \Gamma\left(\frac{b t}{\gamma}\right)\right]^{-a}
$$

which shows power law decay by the exponent $a$ when $a>0$. Note however that the last expression holds for large values of $a$. To sum, the self-referential process for the Gompertz growth model indicates that the derived distribution $F$ has the connection to the quantum statistical distribution as represented in Equation (2) and we shall use it in section 5. In the context of tumour growth, this picture provides a possibility for macroscopic treatments according to the prescription of statistical mechanics.

\section{On Fluctuation of a Growth Parameter}

We consider fluctuation properties (dispersion) of time and a growth rate in this section. Instead of directly dealing with $f(t)$ (Equation (3)) itself, we introduce the velocity $v_{a, b}(t)$ or the change of the number of cells per unit time, which is normalized by the value at infinite time,

$$
v_{a, b}(t) \equiv \frac{d}{d t}\left(\frac{f(t)}{f(\infty)}\right)=a b e^{-b t} e^{-a e^{-b t}}
$$

Then, we find that $v_{a, b}(t)$ satisfies the normalization in the range $[-\infty, \infty]$ with $v_{a, b}(0)=a b / e^{a}$ and reaches a maximum value $b / e$ at $t=\ln a / b$. Therefore, $v_{a, b}(t)$ can be used as a probability distribution over time in order to obtain averaged statistical quantities. The parameter $a$ controls the growth (the regression) rate when the value is positive (negative). On the other hand, the growth parameter $b$ denotes retardation. We recall that the S-shaped curve of the Gompertz growth model depends on the value of the inflection point which is given by $\ln a / 2 b$. Also, the large values of parameter $a$ dominate the shape, which make the point shift rightward. In general, tissues are in different stages of growth and have different velocities depending on their embedded environments and we therefore concern the statistically averaged quantity. Since the velocity defined above is normalized, we can identify $v_{a, b}(t)$ with a weighting distribution function and perform averaging with it. These are the main motivation for employing the velocity distribution for averaging quantities in later discussion. One of the intriguing quantities is the time to get to a certain size. Tissues start growing at various time instants and will reach the steady size or cell numbers according to Gompertz law. Once values for $a$ and $b$ are fixed, the Gompertz curve determines the time to reach a certain level (size or number). Inversely, in case that the sizes at which tumors will arrive differ tumour by tumour and they are statistically distributed in some way, the time will be different and should be averaged over tumors and its dispersion can be calculated for the fixed values of $a>0$ and $b>0$. For the average and the second moment of time, we obtain [16]

$$
\begin{gathered}
\langle t\rangle=\int_{-\infty}^{\infty} t v_{a, b}(t) d t=\frac{\gamma+\ln a}{b} \\
\left\langle t^{2}\right\rangle=\int_{-\infty}^{\infty} t^{2} v_{a, b}(t) d t=\frac{1}{b^{2}}\left((\ln a)^{2}+2 \gamma \ln a+\gamma^{2}+\frac{\pi^{2}}{6}\right)
\end{gathered}
$$

where $\gamma$ is the Euler's constant again. Therefore, the dispersion or the time fluctuation pertain to this growth process is calculated as

$$
\Delta t=\left\langle t^{2}\right\rangle-\langle t\rangle^{2}=\frac{\pi^{2}}{6 b^{2}}
$$


We find that the time fluctuation is determined only by the growth rate $b$. Next, we consider the statistical property of the growth rate $b$. More realistic cases are that $b$ is statistically distributed reflecting the nature of the ensemble of the tumour tissues. Of course, in order to determine the distribution law, we need a detailed dynamics behind the Gompertz model, however it is beyond the present consideration. Without the exact distribution form of $b$, we can move the discussion forward. Then, the moments of the growth rate $b$ are given as

$$
\begin{gathered}
\langle b\rangle=\int_{0}^{\infty} b v_{a, b}(t) d b=\frac{2 a}{t^{3}}{ }_{3} F_{3}[\{1,1,1\},\{2,2,2\},-a] \\
\left\langle b^{2}\right\rangle=\int_{0}^{\infty} b^{2} v_{a, b}(t) d b=\frac{6 a}{t^{4}}{ }_{4} F_{4}[\{1,1,1,1\},\{2,2,2,2\},-a]
\end{gathered}
$$

where ${ }_{p} F_{q}\left(\left\{a_{1}, \ldots, a_{p}\right\},\left\{b_{1}, \ldots, b_{q}\right\} ; z\right)$ is the generalized hypergeometric function defined as

$$
\sum_{k=0}^{\infty} \frac{\left(a_{1}\right)_{k} \cdots\left(a_{p}\right)_{k}}{\left(b_{1}\right)_{k} \cdots\left(b_{q}\right)_{k}} \frac{z^{k}}{k !}
$$

where $z \in \mathbb{R}$ and $(a)_{0}=1,(a)_{k}=a(a+1)(a+2) \cdots(a+k-1)$ for $k \geqslant 1$ being the Pochammer symbol. Assuming $a \simeq 1$, we take the series expansion in $a$

$$
\begin{gathered}
{ }_{3} F_{3}[\{1,1,1\},\{2,2,2\},-a] \simeq 1-\frac{a}{2^{3}}+\left(\frac{1}{3}\right)^{3} \frac{a^{2}}{2 !}-\left(\frac{1}{4}\right)^{3} \frac{a^{3}}{3 !}+\cdots \\
{ }_{4} F_{4}[\{1,1,1,1\},\{2,2,2,2\},-a] \simeq 1-\frac{a}{2^{4}}+\left(\frac{1}{3}\right)^{4} \frac{a^{2}}{2 !}-\left(\frac{1}{4}\right)^{4} \frac{a^{3}}{3 !}+\cdots
\end{gathered}
$$

Thus, the fluctuation of $b$ can be expressed as

$$
\Delta b=\frac{6}{t^{4}} a-\left(\frac{3}{8 t^{4}}-\frac{4}{t^{6}}\right) a^{2}+\left(\frac{1}{27 t^{4}}+\frac{2}{t^{6}}\right) a^{3}-\left(\frac{1}{4^{4} t^{4}}+\frac{91}{432 t^{6}}\right) a^{4}+\cdots
$$

We show in figure 1 the plot of $\Delta b$ as a function of $t$ for some positive values of $a$. The temporal behaviour of $\Delta b$ is decreasing with respect to time, meaning that the fluctuation is suppressing with time.

\section{Entropy Revisited}

The mere growth curve does not convey macroscopic statistical information for processes. Therefore, it is interesting to investigate how the entropy behaves in tumour growth processes, because in a statistical sense, entropy can be a good measure to quantify how order or disorder in a process evolves. We employ the Shannon entropy for this purpose, however, the Gompertz function of the number of cells is a sigmoid curve. This means that the integration of the function in the range $t \in[-\infty, \infty]$ does not converge [17]. This is the main reason which prevents us from obtaining a direct analytical expression for the entropy during the process without computational elaborations if the Gompertz function itself is employed. Moreover, this interval of integration have less biological sense due to the finite time existence of the tumour organisms [18]. To obtain the Gompertzian function as an asymptotic limit in time [6], the entropy concept was useful, where the rate of change of the probability $q(t)$ of quiescent cells in tumour is assumed to be proportional to its binary entropy $-q(t) \log q(t)-(1-q(t)) \log (1-q(t))$. Furthermore, 
to show the superiority of the Gompertzian model over the other two models, i.e., the logistic and the Bertalanffy models, the total entropy change in the entire process of the growth has compared [19], where assumptions and a modification of the integration were required to obtain analytical expressions. There could be several alternatives for defining entropy of the Gompertz growth model. In this section, we point out alternative ways for obtaining the analytic entropy expressions associated with the process. One definition of the entropy is to formulate it as a function of one of the growth parameters. This definition allows us to see the entropy change as a function of time. That is, the expression for the entropy is provided by integrating $-f \log f$ with respect to the growth parameter $a$. For the fixed value of the growth rate $b$ at time $t$, we obtain

$$
S(b, t)=-\int_{0}^{\infty} f_{0} e^{a\left(1-e^{-b t}\right)}\left[\log f_{0}+a\left(1-e^{-b t}\right)\right] d a
$$

If $b<0$, we obtain the analytical expression by performing the integration

$$
S(b, t)=\frac{a f_{0} \sinh (b t)-\frac{f_{0}}{2} \log f_{0}\left(1-e^{b t}\right)}{1-\cosh (b t)}
$$

This expression implies that the initial higher entropy state asymptotically converges to a constant value. We plot this behaviour for some different values of $b$ in Figure 2.

Figure 1. The comparison of the decreasing behaviour of the dispersion for the growth rate $b$ for two cases, i.e., $a=0.4$ (the solid line) and $a=0.2$ (the broken line) according to Equation (17) up to the 4th oder in $a$.

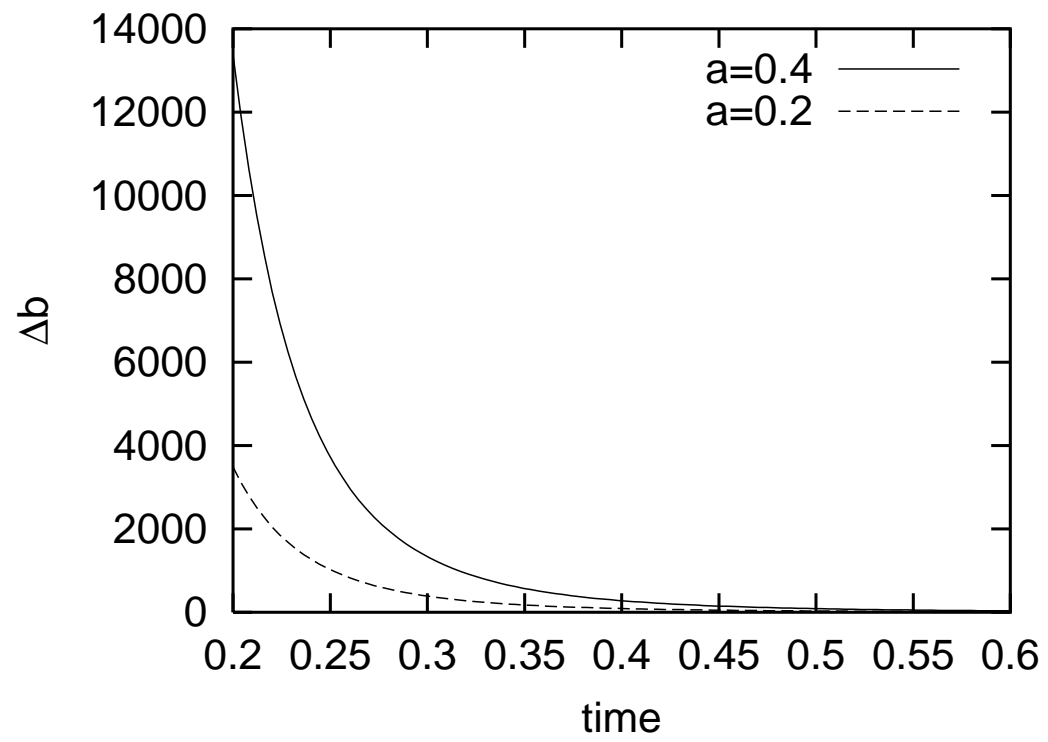

The other definition that we are concerned with is based on the velocity or the rate of change $v_{a, b}(t)=a b e^{-b t} e^{-a e^{-b t}}$ used in the previous section. To see the time evolution of the entropy for the growth velocity, we introduce $w_{a, b}(t)$, the growth velocity normalized by the initial velocity $v_{a, b}(0)$ and integrate a quantity $-w_{a, b}(t) \log w_{a, b}(t)$ with respect to $a$ in the range $[0, \infty]$. Then, we have an analytical 
Figure 2. The behaviour of entropy $S(b, t)$ given by Equation (19) as a function of time for $b=-0.5$ and $b=-2.0$. We set $a=1.0$ and $f_{0} \log f_{0}=1.0$.

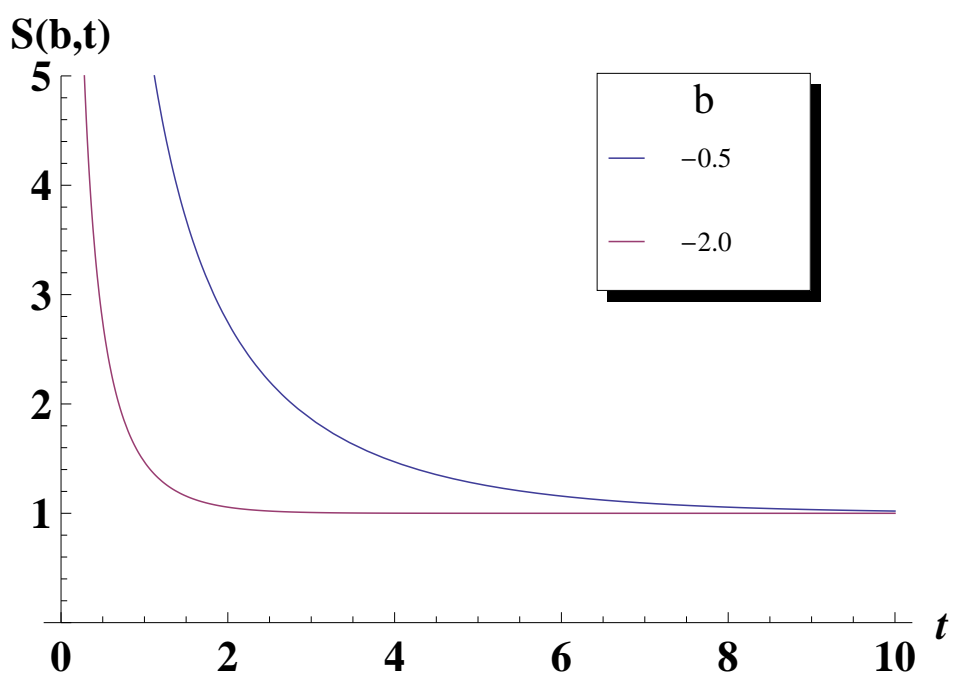

expression if $b<0$,

$$
\begin{aligned}
S(b, t) & =-\int_{0}^{\infty} e^{a-b t} e^{-a e^{-b t}}\left[a-b t-a e^{-b t}\right] d a \\
& =\frac{1+b t}{1-e^{b t}}
\end{aligned}
$$

This entropy decreases with time and reaches zero, which corresponds to the asymptotic approach to the vanishing growth velocity and can be regarded reasonable in a sense that it matches the Gompertz sigmoidal curve. Once the system reaches the plateau in the curve, the entropy will never change. Figure 3 shows the behaviour of Equation (20) for two different values of growth rates $b$. For other possible choices (e.g., $S(a, b)$ and $S(a, t)$ etc.) in the framework of the logarithmic entropy, we cannot obtain analytical expressions. These characterization of the Gompertz law as a probability function encourage us to focus on the comparison of the role of the two growth rates $a$ and $b$ in the statistical description. In general, growth parameters $a$ and $b$ can be related each other depending on the environment that tissues are embedded, but they are tuned independently in terms of the data fitting. If $a$ and $b$ are not independent variables, there should be underlying laws, however, specifing them is beyond our present scope. One of the meaningful quantities in the ensemble analysis would then be a ratio of the averaged values. We define the following ratio $r$ [20] for a quantity $g$

$$
r:=\frac{\langle g\rangle_{f(a, b)}}{\langle g\rangle_{f(b, a)}}
$$

where $g$ denotes any function of $a$ and $b$, not depending on time. The averages are taken with the functions $f(a, b)$ and $f(b, a)$, respectively. The Gompertz function is not symmetric under the change of the two growth parameters, i.e, $f(a, b) \neq f(b, a)$. This implies that the ratio should contain the statistical information for the role of the two growth parameters. Performing the integration, we obtain

$$
r=\frac{a}{b} e^{a-b} \frac{\Gamma(0, a)}{\Gamma(0, b)}
$$


Figure 3. The behaviour of entropy $S(b, t)$ given by Equation (20) as a function of time for $b=-0.5$ and $b=-2.0$ when $a=1.0$ and $f_{0} \log f_{0}=1.0$.

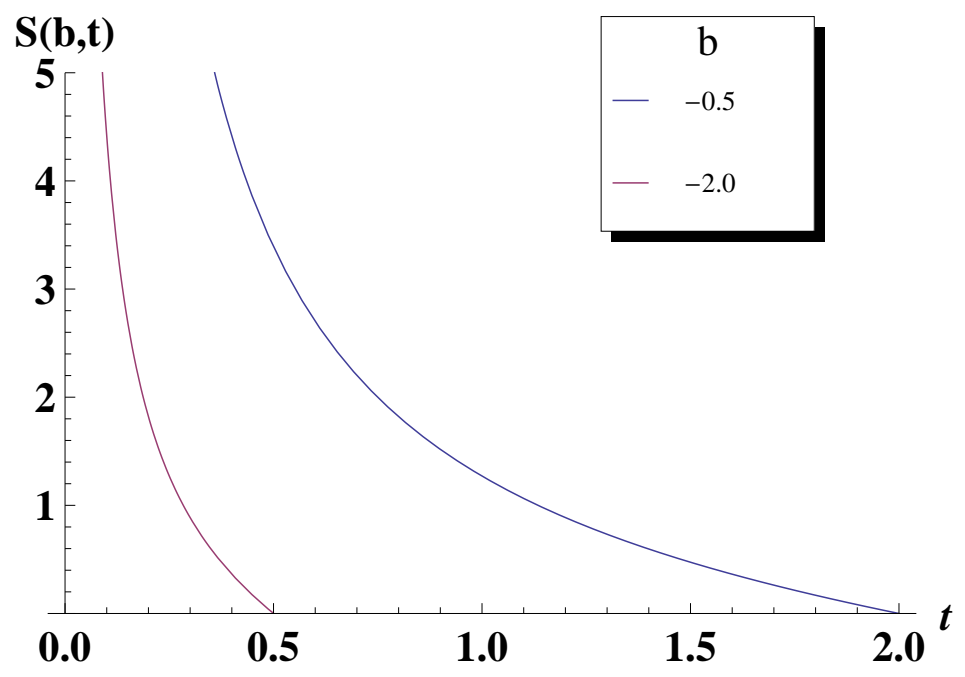

where $\Gamma(a, z)=\int_{z}^{\infty} t^{a-1} e^{-t} d t$ is the incomplete gamma function. When the two growth rates take the same value, $r=1$ holds. In this sense, the deviation from the unity would provide the estimation of the statistical weight on the growth process. We plot the $r$ as functions of $a$ and $b$ in Figure 4.

Figure 4. The ratio of two statistical averages of function $g$ given by Equation (22). It diverges as both $a$ and $b$ approach zero.

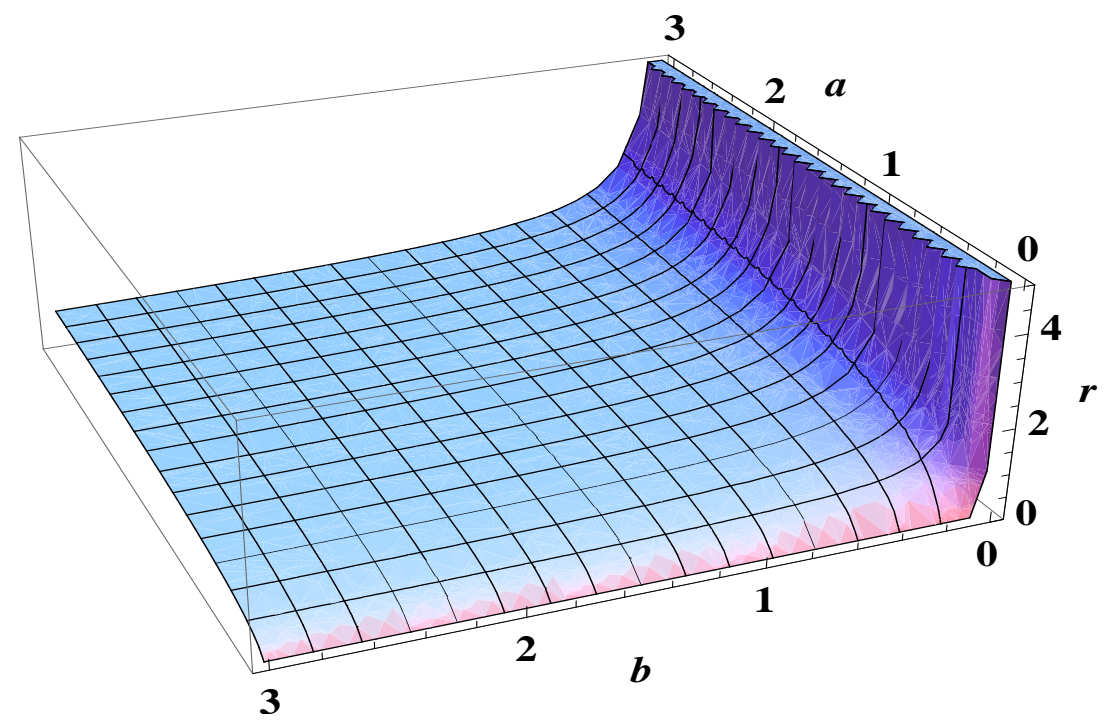


Next, we consider the ratio of the averaged value of the factor $e^{-a t}$ and that of $e^{-b t}$. We define the following simultaneous ratios $r_{1}$ and $r_{2}$ of the averaged growth factors as follows,

$$
r_{1}=\frac{\left\langle e^{-a t}\right\rangle_{f}}{\left\langle e^{-b t}\right\rangle_{f}} \quad r_{2}=\frac{\left\langle e^{-a t}\right\rangle_{v}}{\left\langle e^{-b t}\right\rangle_{v}}
$$

where $\langle\cdot\rangle_{f}$ and $\langle\cdot\rangle_{v}$ denote averages over $t \in[0, \infty]$ with the function $f(t)$ and with the velocity $v_{a, b}(t)$, respectively. We think that the choice of this integration interval does not alter the results due to the exponential function of the integrand even when a finite time is set. Note also that these are ratios of the Laplace transform of the Gompertz function and its derivative, respectively. When $\operatorname{Re}(\mathrm{a})>0$ and $\operatorname{Re}(\mathrm{b} / \mathrm{a})>0$, we have the expression

$$
r_{1}=\frac{a^{1-\frac{b}{a}}\left[\Gamma\left(\frac{b}{a}\right)-\Gamma\left(\frac{b}{a}, a\right)\right]}{1-e^{-a}}
$$

and when $\operatorname{Re}(\mathrm{a})>0$ and $\operatorname{Re}(\mathrm{a} / \mathrm{b})>-1$, we have the expression

$$
r_{2}=\frac{a^{1-\frac{a}{b}} e^{a}\left[\Gamma\left(1+\frac{a}{b}\right)-\Gamma\left(1+\frac{a}{b}, a\right)\right]}{e^{a}-1-a}
$$

where $\Gamma(z)$ is the gamma function. If the two growth rates are the same, i.e., $a=b$, then $r_{1}=r_{2}=1$ holds by necessity. In Figures 5 and 6, we show their behaviour as functions of $a$ and $b$, respectively. The Figure 5 indicates that there is a line on $a-b$ plane, where the $r_{1}$ value becomes zero and for

Figure 5. The plot of the ratio $r_{1}$ given by Equation (24) as a function of $a \in[0,1]$ and $b \in[0,1]$.

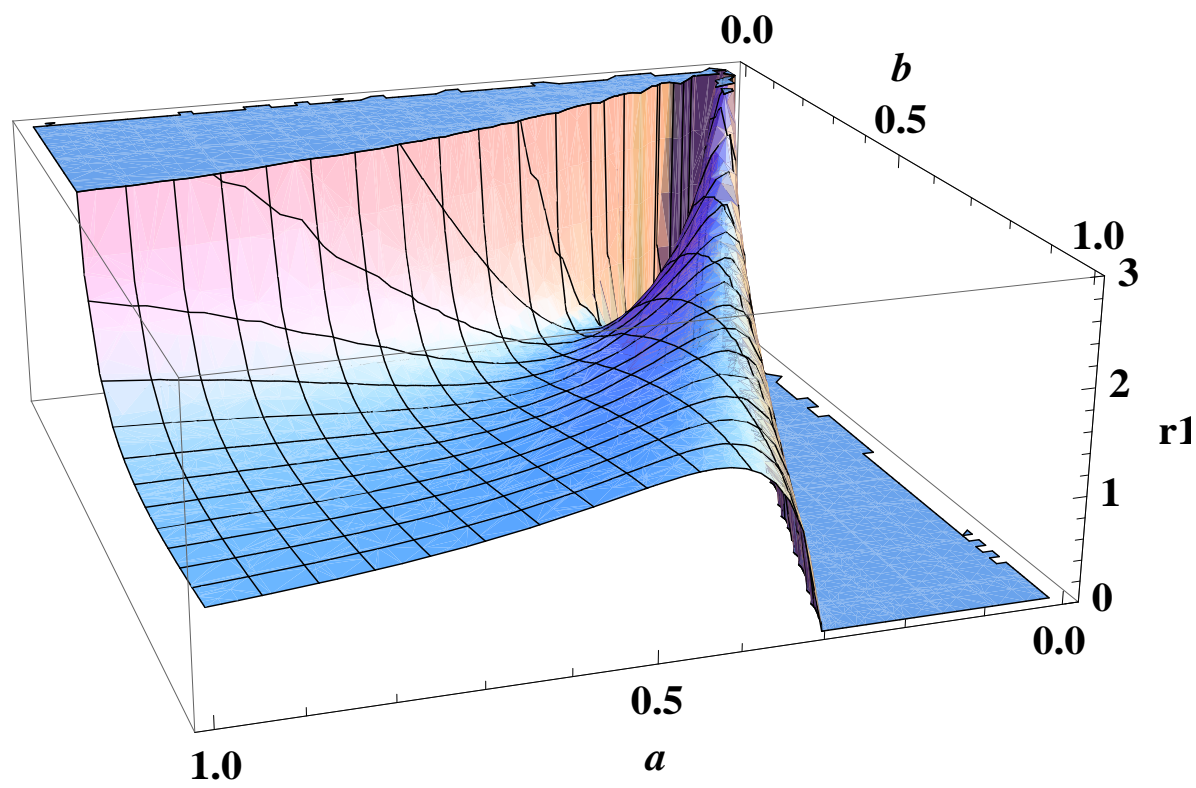

small values of $b, r_{1}$ tends to diverge, i.e., the averaged factor of $e^{-a t}$ is much larger than that of $e^{-b t}$. This can be interpreted as indicating that the growth parameter $b$ is statistically dominating compared to $a$. Likewise, the divergent behaviour can be seen in figure 6, which enables us to have statistically 
significant interpretation, i.e., the domination of $a$ (or $b$ ) over $b$ (or $a$ ) depending on their values. In the context of tumour cell ensemble, these facts indicate that the growth parameters $a$ and $b$ statistically control the progress and retardations of growth.

Figure 6. The plot of the ratio $r_{2}$ given by Equation (25) as a function of $a \in[0,3]$ and $b \in[-3,0]$.

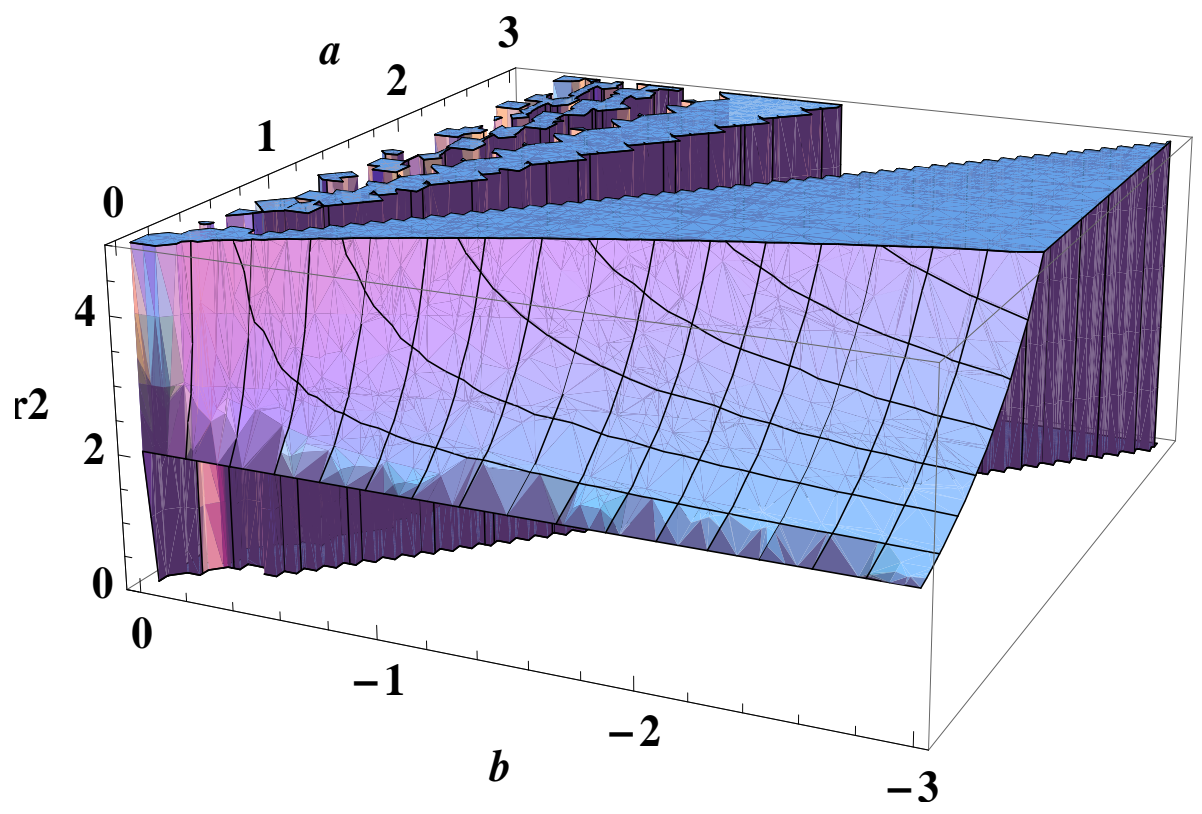

\section{Bose-Einstein Condensation Picture for the Gompertz Model}

We have seen in Section 2 that the self-replication process in terms of the distribution function for the Gompertz model provides the Bose-Einstein statistics form, which was originally introduced to describe statistics for photons and atoms [21]. We shall further pursue this remarkable similarity to this statistics. We restrict our consideration to the case that the growth dynamics is regressive i.e., $a<0$ to keep this analogy and the tumour cells are indistinguishable. As we mentioned in Section 2, the case $a>0$ amounts to deal with the logistic model and does not pose new challenges driven by this similarity. Furthermore, let us assume that the parameter $a$ is discretized and put it as

$$
c_{l}=-a_{l}=\epsilon+d_{l} \quad \epsilon \geq 0 \quad(l=0,1, \cdots, \infty)
$$

where the constant $\epsilon$ is the lowest value of $c_{l}$ and the parameter $d_{l}$ bears the discreteness with $d_{0}=0$. From Equation (4), we define the mean number of tumour cells $\left\langle n_{l}\right\rangle$ in an organism and suppose that the organism lies in the state level $l$, then

$$
\left\langle n_{l}\right\rangle=\frac{1}{e^{c_{l} q}-1} \quad(l=0,1, \cdots, \infty)
$$

where $q \equiv 1-e^{-b t}$ takes the range $0 \leq q \leq 1$ corresponding to the time range $0 \leq t \leq \infty$ for a fixed value of $b$. Then, the total number $N$ is written as

$$
N=\sum_{l=0}^{\infty}\left\langle n_{l}\right\rangle=\frac{1}{e^{\epsilon q}-1}+\sum_{l=1}^{\infty} \frac{1}{\eta^{-1} e^{q d_{l}}-1}
$$


where we have introduced a variable $\eta \equiv e^{-\epsilon q}, \quad\left(e^{-\epsilon} \leq \eta \leq 1\right)$. We evaluate the second term of Equation (28). As is often the case in natural sciences, the density of states $\omega$ of density $y$ takes the form of a power law,

$$
\omega(y)=\text { const. } \times y^{s}, \quad s \geq 0
$$

Then, the second term can be replaced as a continuous form,

$$
\int_{0}^{\infty} \frac{\omega(y)}{\eta^{-1} e^{q y}-1} d y=\text { const. } \times q^{-s-1} \Gamma(s+1) \phi(s+1, \eta)
$$

where $\phi(s, \eta)$ is the Appel function defined as

$$
\phi(s, \eta)=\frac{1}{\Gamma(s)} \int_{0}^{\infty} \frac{x^{s-1}}{\eta^{-1} e^{x}-1} d x
$$

As this integration of the second term excludes the state of $y=0$, the division of the number $N$ into two parts in Equation (28) is consistent. Now, we set a critical value $q_{c}$ when $\eta$ takes unity (i.e., when $\epsilon=0$ ), then we have an expression,

$$
N=\text { const. } \times q_{c}^{-s-1} \Gamma(s+1) \phi(s+1,1)
$$

Therefore $N$ is written as

$$
N=\frac{\eta}{1-\eta}+N\left(\frac{q}{q_{c}}\right)^{-s-1} \frac{\phi(s+1, \eta)}{\phi(s+1,1)}
$$

Since $q$ and $\eta$ are not independent variables each other (they are related by $q=-\frac{\ln \eta}{\epsilon}$ ), we can write

$$
\frac{N_{0}}{N}=1-\left(\frac{\ln \eta}{\ln \eta_{c}}\right)^{-s-1} \frac{\phi(s+1, \eta)}{\phi(s+1,1)}
$$

where $N_{0}=\eta /(1-\eta)$ is the number of states for the level $l=0$. This means that when $\eta$ satisfies $\ln \eta \leq \ln \eta_{c}, N_{0} / N=0$ holds and when $\ln \eta \geq \ln \eta_{c}$, we have a form at $\eta \simeq 1$

$$
\frac{N_{0}}{N}=1-\left(\frac{\ln \eta}{\ln \eta_{c}}\right)^{-s-1}
$$

The existence of the critical value in $\eta$ means that there is a corresponding critical value in the growth rate $b_{c}$ at a certain finite time, which is given by

$$
b_{c}=-\ln \left(1+\frac{\ln \eta_{c}}{\epsilon}\right) / t
$$

For this value, the number of the lowest state $N_{0}$ becomes large as $N$ becomes large, which means the Bose-Einstein condensation. We have seen that the straightforward application of the Bose-Einstein statistics was feasible due to the equivalent form of the Gompertz type distribution function to this statistics for occupation number. In the context of tumour population, this implication shows that all the indistinguishable cells tend to herd at the same lowest state to form a condensate of the tumour tissue. On the other hand, in the case of another quantum statistical similarity (with Fermi-Dirac statistics), the average number takes between 0 and 1 (occupied or empty for each energy level), which may not match our present consideration in tumour context, although calculations can be performed according to a textbook on statistical mechanics (e.g., [22]). 


\section{Summary and Concluding Remarks}

We have discussed the ensemble approach to the Gompertz type growth model, where two growth parameters are supposed not to be single-valued for all elements but are statistically distributed. This setup enabled us to consider the statistical mechanics features in the context of tumour cells. Distribution functions are main ingredients for the discussion of statistical treatments of systems. The self-referential process in Equation (1) for the distribution function has two possibilities, i.e., the self-replication and the self-feedback. We have shown that if we apply the self-replication process to the Gompertz growth within the domain $b t \ll 1$, a logistic growth law is included in the generated distribution function. The causal dynamics producing the Gompertz form is at present not fully clear in the literature. However, we have shown that a statistical ensemble approach can be performed. In terms of the growth velocity function, the fluctuation of the growth rate $b$ can be expressed $\Delta b=\sum_{k=1}^{\infty} g(t) a^{k}$, where $g(t)$ is determined from the coefficients of the generalized hypergeometric functions. As long as the growth state is in a regressive phase $(a<0)$, we can keep the Bose-Einstein statistics description. This would motivate us for directions for further investigation in quantum statistical setting. In fact, there is a recent desire for the exploratory power of quantum theory to mechanisms behind biological phenomena [23, 24]. In this perspective, an exemplification for the connection between the Gompertz function and a quantum coherent state is also intriguing, where the Gompertz functional form can be regarded as a solution of a time-dependent counterpart of the Schrödinger equation for the Morse oscillator with the anharmonicity constant equal to unity [25]. In tumour growth context, the direct comparison of the present approach and obtained formulae with real experimental data set of tumors remains future work.

\section{Acknowledgements}

The author is indebted to the department seminar given by Prof. Y. Iwasa on mathematical biology during 27-28 February 2009 and to his feedback at the department of physics, Ochanomizu university, Tokyo.

\section{References and Notes}

1. Gompertz, B. On the nature of the function expressive of the law of human mortality, and on a new mode of determining the value of life contingencies. Phil. Trans. Roy. Soc. 1825, 115, 513-585.

2. Casey, A.E. The experimental alteration of malignancy with an homologous mammalian tumour material I. Am. J. Cancer 1934, 21, 760-775.

3. Bajzer, Z.; Vuk-Pavlovic, S. Mathematical modelling of tumor growth kinetics. In A Survey of Models for Tumor-Immune System Dynamics; Adam, J.A., Bellomo, N., Eds.; Birkhauser Publishing: Basel, Switzerland, 1997; pp. 89-133.

4. Bajzer, Z. Gompertzian growth as a self-similar and allometric process. Growth Develop. Aging 1999, 63, 3-11.

5. Kendal, W.S. Gompertzian growth as a consequence of tumor heterogeneity. Math. Biosci. 1985, 73, 103-107.

6. Calderón, C.P.; Kwembe, T.A. Modelling tumor growth. Math. Biosci. 1991, 103, 97-114. 
7. Gunasekaran, N.; Pande, L.K. Log normal distribution for the intrinsic abundance for species from the Gompertz model. J. Theor. Biol. 1982, 98, 301-305.

8. Sitaram, B.R.; Varma, V.S. Statistical mechanics of the Gompertz model of interacting species. $J$. Theor. Biol. 1984, 110, 253-256.

9. Castorina, P.; Zappala, D. Tumor Gompertzian growth by cellular energetic balance. Physica A 2006, 365, 473-480.

10. Lo, C.F. Stochastic model of tumour cell growth. J. Theor. Biol. 2007, 248, 317-321.

11. Waliszewski, P.; Konarski, J. On time-space of nonlinear phenomena with Gompertzian dynamics. Biosystems 2005, 80, 91-97.

12. Waliszewski, P. A principle of fractal-stochastic dualism and Gompertzian dynamics of growth and self-organization. Biosystems 2005, 82, 61-73.

13. Apostol, B.-F. Euler's transform and a generalized Omori's law. Phys. Lett. A 2005, 351, 175-176.

14. Swan, G.W. Role of optimal control theory in cancer chemotherapy. Math. Biosci. 1990, 101, 237-284.

15. Kuznetsov, V.A.; Makalkin, I.A.; Taylor, M.A.; Perelson, A.S. Nonlinear dynamics of immunogenic tumors: Parameter estimation and global bifurcation analysis. Bull. Math. Biol. 1994, 56, 295-321.

16. We have used Mathematica 7 here and also for later calculations of integrations.

17. If the interval $t \in[0, \infty]$ is set, the integration becomes $-f_{0} e^{a} \Gamma(0, a) / b$ when $a>0$ and $b<0$.

18. A way out is to introduce a cut-off time or a death time of tumour cells, however we need dynamics to determine the value. Therefore we do not elaborate this point in this paper.

19. Ling, Y.; He, B. Entropic analysis of biological growth models. IEEE Trans. Biomed. Eng. 1993, 40, 1193-1200.

20. Note that $r$ is different from the proliferation rate in Equation (1).

21. The number of indistinguishable particles $n_{i}$ in the energy $E_{i}$ is given by $n_{i}=\frac{1}{e^{\beta\left(E_{i}-\mu\right)}-1}$ when there is no degeneracy, where $\beta$ is the inverse temperature and $\mu$ the chemical potential.

22. Pathria, R.K. Statistical Mechanics, 2nd ed.; Butterworth-Heinemann: Oxford, UK, 1996.

23. Abbott, D.; Davis, P.C.; Pati, A.K. Quantum Aspect of Life; Imperial College Press: London, UK, 2008.

24. Davies, P. A quantum recipe for life. Nature 2005, 437, 819.

25. Molski, M.; Konarski, J. Coherent states in Gompertzian growth. Phys. Rev. E 2003, 68, 21916-21922.

(c) 2009 by the authors; licensee Molecular Diversity Preservation International, Basel, Switzerland. This article is an open-access article distributed under the terms and conditions of the Creative Commons Attribution license (http://creativecommons.org/licenses/by/3.0/). 